REVIEW ARTICLE

\title{
Imaging for Sensorineural Hearing Loss
}

\section{Santosh Gupta}

Department of Radiology, PD Hinduja National Hospital and Medical Research Center, Mahim, Mumbai, Maharashtra, India

Correspondence: Santosh Gupta, Department of Radiology, PD Hinduja National Hospital and Medical Research Center Veer Savarkar Marg, Mahim, Mumbai-400016, Maharashtra, India

\section{Abstract}

There are several causes of sensorineural hearing loss (SNHL), in some of which imaging plays an important role for diagnosis and further management. We present an elaborate imaging based pictorial depiction of the various findings in these cases mainly based on $\mathrm{CT}$ and $\mathrm{MRI}$ imaging.

Keywords: Sensorineural hearing loss, imaging, CT, MRI.

\section{INTRODUCTION}

Diagnostic imaging plays an important role in the evaluation and management of hearing loss. High resolution computed tomography (CT) of the temporal bone and magnetic resonance imaging (MRI) are the imaging modalities of choice to evaluate the ear structures for causes of hearing loss and are now routinely being performed. Older imaging techniques of plain radiographs or X-ray tomograms are now obsolete. Also in general, to evaluate the bony structures, including the external auditory canal, middle ear space, mastoid, petrous apex, and bony labyrinth CT is a better modality, whereas for the membranous labyrinth and suspicion of retrocochlear pathology MRI should be the imaging modality of choice. ${ }^{1}$ However, many a times both modalities are used together to compliment each other. MRI with its higher soft tissue resolution and tissue characterization capabilities would be more frequently used in cases of sensorineural hearing loss (SNHL), where the pathology is generally in the cochlea or retrocochlear region (in the eight nerve or brain).

\section{IMAGING TECHNIQUE}

It is vital to obtain a good quality high resolution CT and MRI, in patients with SNHL, with accurate technical paramaters. Most centers are now equipped with multislice/ spiral CT scanners, which is essential as reconstructions can be obtained in any plane with isotropic voxels in high end CT scanners without loss of resolution. MRI should be obtained on a high tesla strength magnet, 1T (tesla) or higher. The author's experience in his institute, is on a 64 slice spiral CT and 1.5 T MRI machine, details of which have been elaborated below:

\section{HRCT}

HRCTs on a 64 slice volume scanner (LightSpeed VCT, GE, Milwaukee, USA) are obtained in a straight axial plane $(\mathrm{kV}: 140$, mA: 350, matrix: $512 \times 512$, slice thickness: $0.625 \mathrm{~mm} / 10.63,0.531: 1$, scan field of view (FOV): $32 \mathrm{~cm}$, display FOV: $9.6 \mathrm{~cm}$ ). The original isometric volume data is used to obtain coronal reformatted images. The images are then reviewed with a high resolution bone algorithm, using a small FOV for separate right and left ear documentation.

MRI

MRI scans on 1.5 T MR machine (Excite Twin Speed, GE, Milwaukee, USA) are performed with an 8-channel head coil. A routine $\mathrm{T} 2 \mathrm{~W}$ axial sequence through the brain is obtained in all patients. For evaluating the seventh-eighth nerve complex thin $3 \mathrm{~mm}$ slices with 0 spacing are obtained through the posterior fossa in axial and coronal planes, both plain and following intravenous administration of gadolinium contrast. Diffusion weighted sequence is also obtained through the brain.

In addition, especially for evaluation of nerves and in patients posted for cochlear implantation a 3D-FIESTA (fast imaging enabling steady-state acquisition) axial sequence (TR: 5.5, TE: 1.7/Fr, FOV: $16 \times 16$, slice thickness: 1.0/ -0.5 , matrix: $320 \times 320$, NEX: 6.0 ) is performed followed by coronal reformations along with $3 \mathrm{D}$ maximum intensity 
projection (MIP) reconstructions. A 3D-FIESTA sequence is also acquired in a direct oblique sagittal plane (TR: 6.7, TE: 2.1/Fr, FOV: $12 \times 12$, slice thickness: 1.0/- 0.5, matrix: $384 \times 320$, NEX: 6.0 ) perpendicular to the VII-VIII nerve complexes. With this technique we are able to obtain better resolution than with reformations from an axial sequence; which enables better delineation of the nerves.

\section{IMAGING FINDINGS BASED ON ETIOLOGIES OF SNHL}

The etiologies of SNHL where imaging plays an important role can be broadly classified into congenital and acquired. Congential causes include various vestibulo-cochlear malformations, congenital syndromes and intrauterine infections. Acquired causes include inflammatory/infective, trauma, tumors, vascular ischemia, otodystrophies. ${ }^{2}$ Many of the other causes of SNHL such as ototoxic drug-induced, noise-induced, autoimmune, idiopathic, etc are not likely to show any significant findings on imaging and therefore in these cases, imaging has a limited role to play, mainly to exclude other above imaging-discernible etiologies.

To discuss imaging of all the varied etiologies of SNHL is beyond the scope of this article. Therefore relevant cases, which are commonly encountered have been discussed below.

\section{Congenital Malformations}

Based on the site of abnormality, congenital inner ear anomalies can be classified into:

a. Cochlear malformations

b. Vestibular malformations

c. Malformations of the semicircular canals

d. Vestibular and cochlear aqueduct malformations

e. Cochlear nerve deficiency

f. Isolated attenuated modiolus, and

g. Isolated cochlea. A classification commonly used by ENT surgeons is the one described by Sennaroglu and Saatci; ${ }^{3}$ we have used this system with some modifications, since we encountered some additional anomalies on MRI (such as cochlear nerve deficiency). ${ }^{4}$ Congenital malformations of the inner ear are rare anomalies; they can be identified on imaging with HRCT and/or MRI in about $20 \%$ of patients with congenital sensorineural hearing loss. ${ }^{5}$

With the resolution provided by the newer CT scan and MRI equipment it is now possible to see minute internal structures of the cochlea such as the interscalar septum, ${ }^{6}$ which divides the major cochlear turns; this can be seen on both HRCT and MRI 3D-FIESTA sequences (Fig. 1A). The osseous spiral lamina, a thin membrane within each turn of the cochlea that separates the scala vestibuli from the scala tympani, ${ }^{6}$ can also be well seen on MRI as a thin, linear structure. The MRI 3D sequence data can be used to obtain a 3D MIP reconstruction (Fig. 1B), which gives a good outline of the inner ear structures, especially when complex anomalies need evaluation. The membranous

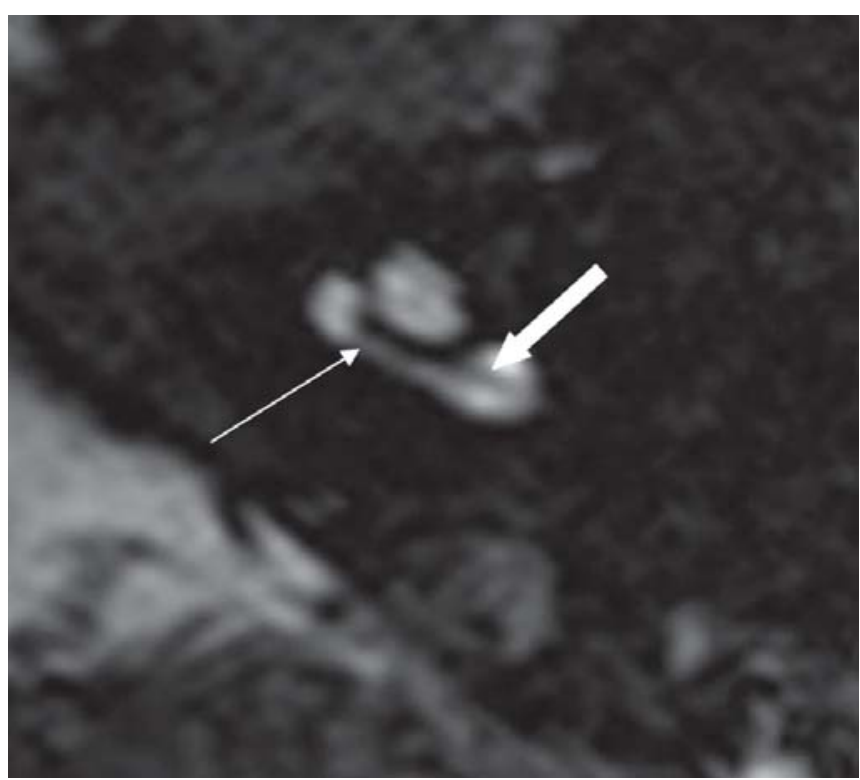

Fig. 1A: Axial 3D-FIESTA sequence, at the level of cochlea shows interscalar septum (arrow) and osseous spiral lamina (block arrow)

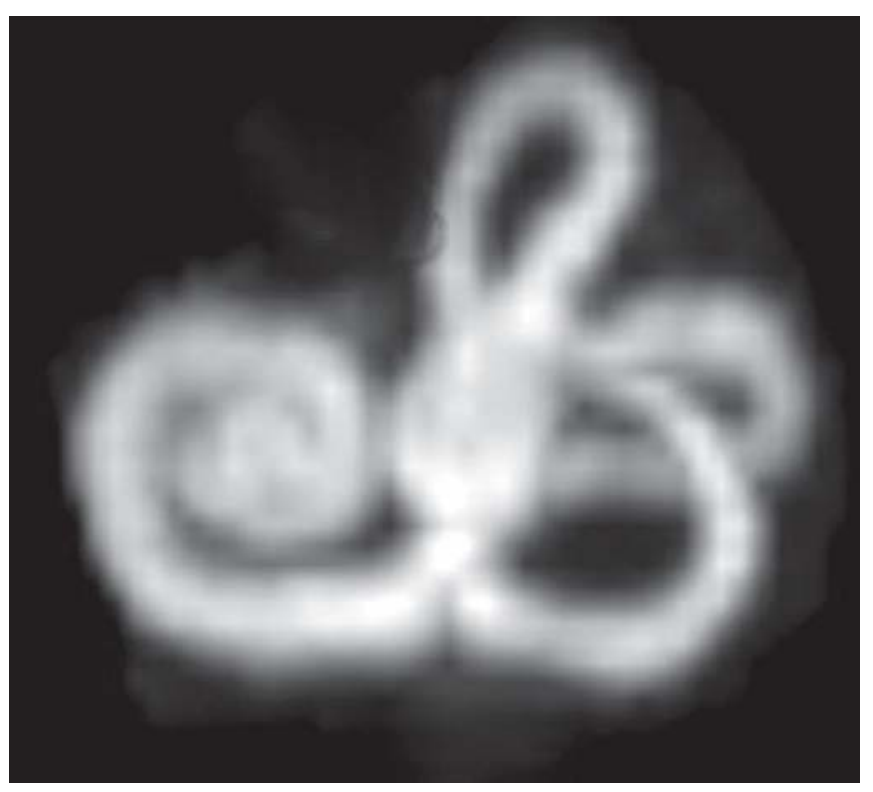

Fig. 1B: 3D-MIP reconstruction of the data from 3D-FIESTA sequence shows the anatomy of the membranous labyrinth 
labyrinth contains endolymph and is surrounded by the perilymph which, in turn, separates it from the otic capsule or bony labyrinth. The cochlea consists of two and one half turns, which extend into the vestibule. The three semicircular canals arise from the vestibule in arches along all three planes.

The vestibular aqueduct represents an osseous aperture in the bony labyrinth; it is about $5 \mathrm{~mm}$ in length and is located along the medial aspect of the pyramid. Although, its lumen is lined by squamous and cuboidal epithelium, it houses an extension of the membranous labyrinth-the endolymphatic duct. On axial CT scan, the vestibular aqueduct is seen as a small slit running medial and parallel to the plane of the posterior semicircular canal.

The seventh-eighth nerve complexes are well seen on MRI. On the 3D-FIESTA sequence it is possible to identify further divisions of the eighth nerve (Fig. 2A). The cochlear nerve is well seen on axial 3D-FIESTA images (Fig. 2B), extending into the modiolus. The anatomy and delineation of the nerves is better appreciated in an oblique sagittal plane, perpendicular to the plane of the internal auditory canal. ${ }^{7}$ With this technique (Fig. 2C), the superior and inferior vestibular nerves are seen in the posterior quadrants and the cochlear nerve in the anteroinferior quadrant, while the facial nerve is seen in the anterosuperior quadrant.

The important congenital anomalies that are encountered when imaging patients prior to cochlear implant surgeries, are discussed in detail below.

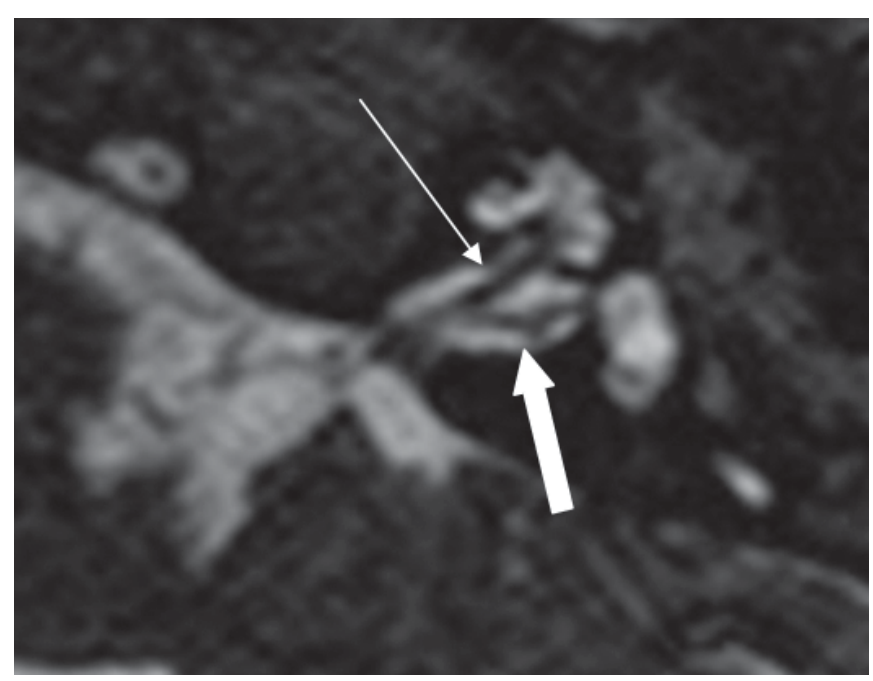

Fig. 2A: Axial 3D-FIESTA sequence, at the level of vestibule and cochlea shows cochlear nerve (arrow) and vestibular nerve (block arrow)

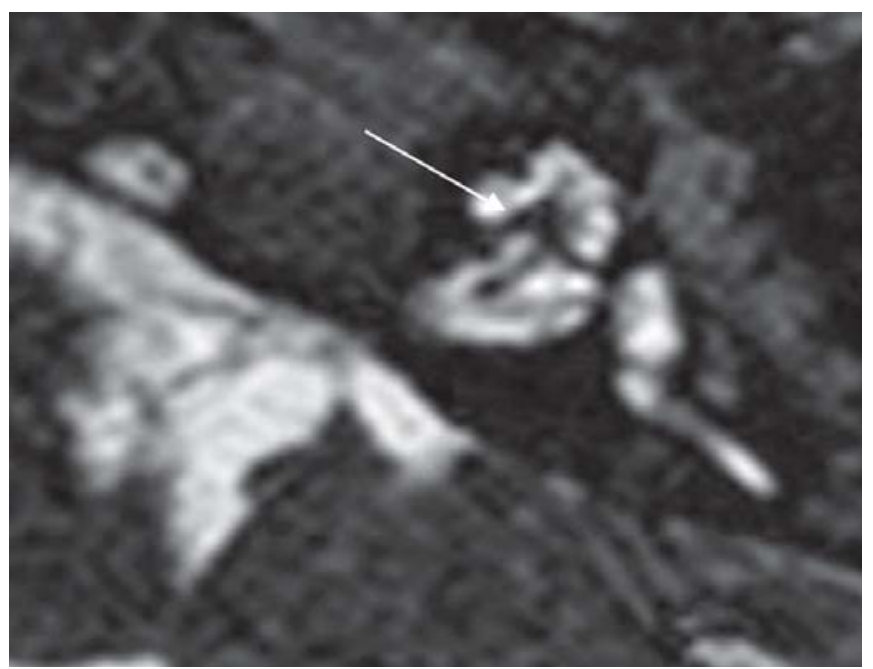

Fig. 2B: Axial 3D-FIESTA sequence, at the level of vestibule and cochlea, shows well-delineated and intact modiolus (arrow)

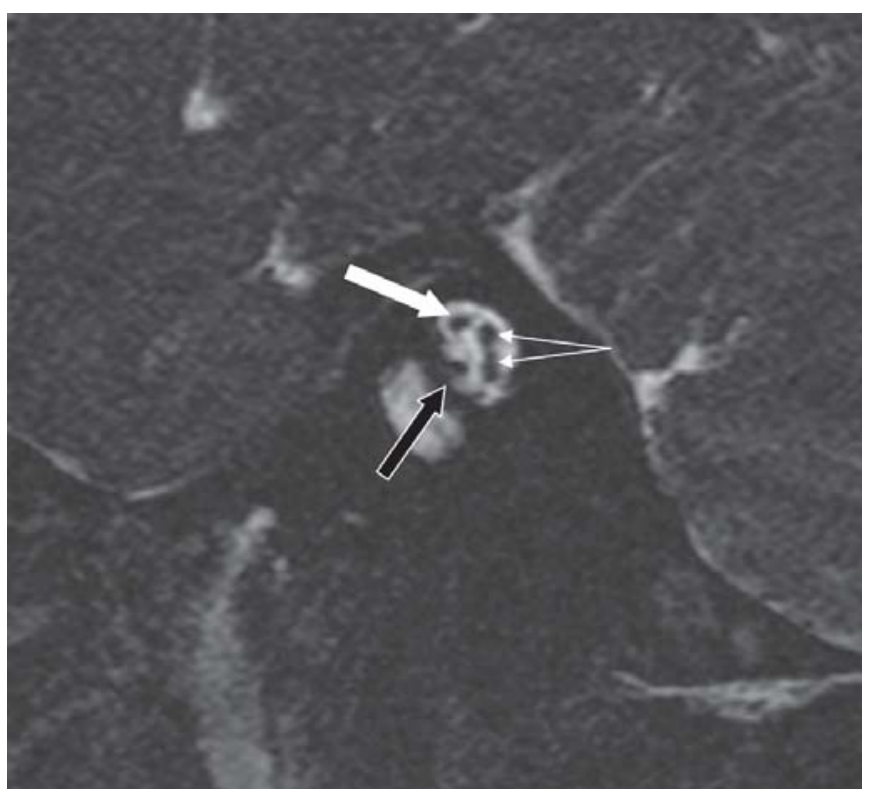

Fig. 2C: Oblique sagittal 3D-FIESTA sequence through the internal auditory canal shows facial nerve in the anterosuperior quadrant (block white arrow), cochlear nerve in the anteroinferior quadrant (block black arrow) and the superior and inferior vestibular nerves, in the posterior quadrant (branching arrows)

\section{Michel Deformity}

In this deformity, there is absence of the entire cochlea and the vestibular structures, i.e. complete labyrinthine aplasia. ${ }^{3}$ It may be bilateral or unilateral (Fig. 3). The internal auditory canals (IACs) are small in size on both sides (Figs 3A and B). Cochlear nerve deficiency may be seen (Fig. 3C), on MR 3D-FIESTA images. 


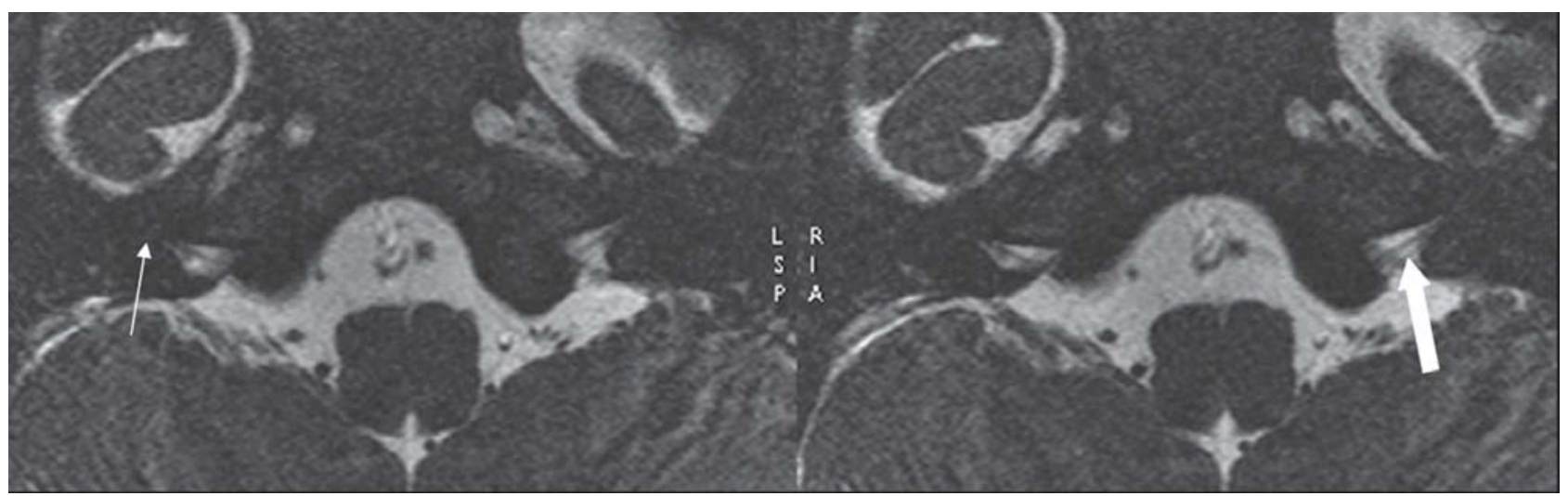

Fig. 3A: Axial 3D-FIESTA sequence shows absence of the entire vestibulo-cochlear structures, bilaterally (arrow), suggesting Michel deformity. The internal auditory canals are small on both sides, with markedly thin eighth nerve (block arrow on left side)

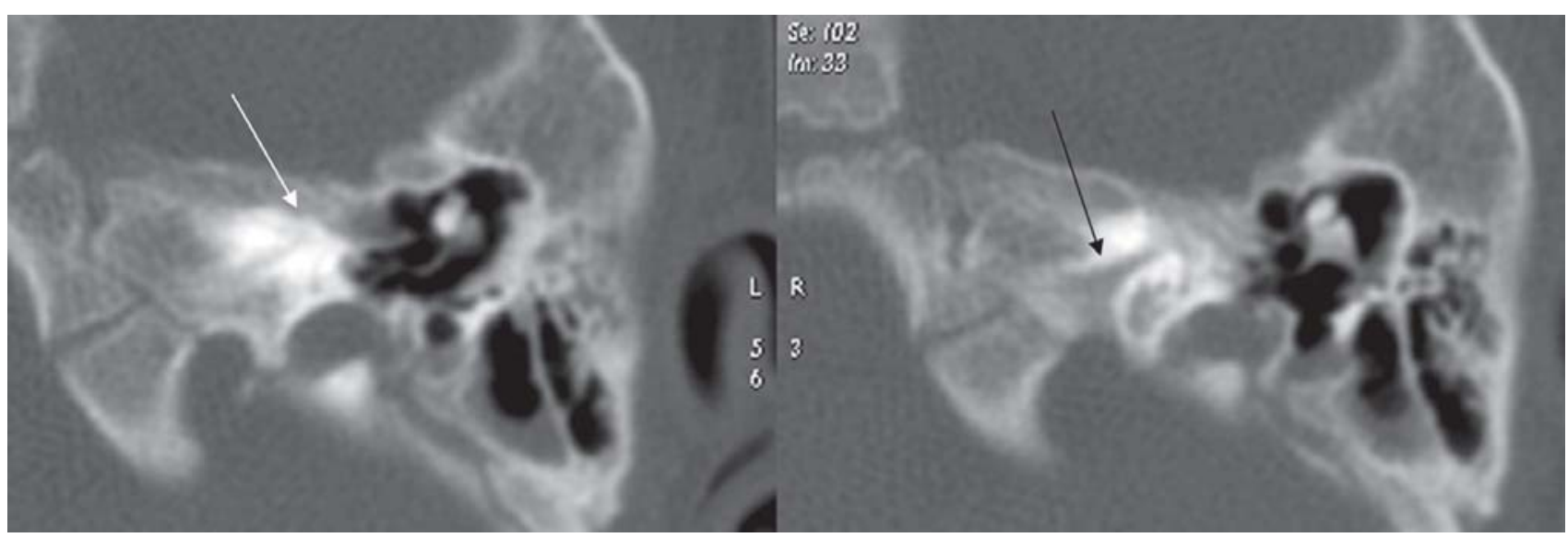

Fig. 3B: HRCT shows small left internal auditory canal (black arrow) and absence of the entire vestibulo-cochlear structures (white arrow), consistent with Michel deformity

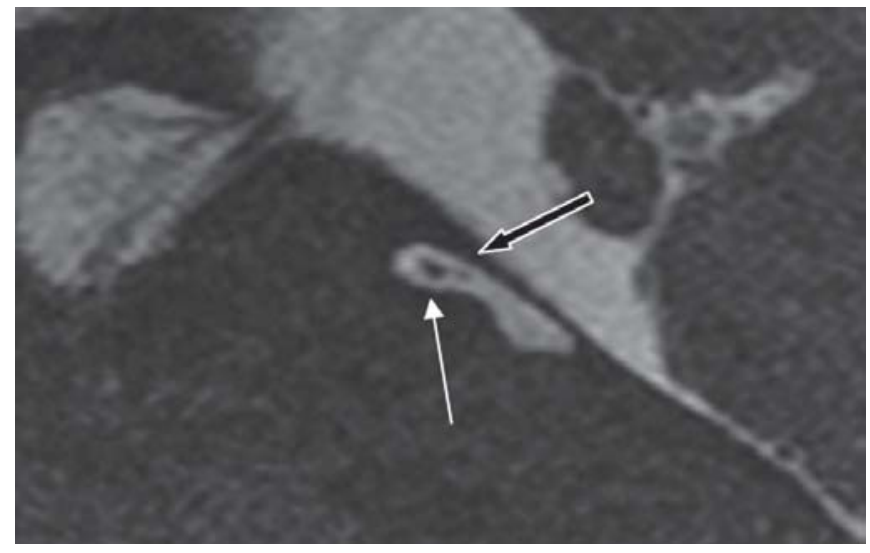

Fig. 3C: Oblique sagittal 3D-FIESTA sequence, through the small internal auditory canal shows thinned-out eighth nerve (block back arrow), with no divisions and normal facial nerve antero-superiorly (arrow)

\section{Cochlear Aplasia}

In this condition, the cochlea is completely absent ${ }^{3}$ (Fig. 4). The vestibule and semicircular canals may be normal, dilated (Figs 4A and B), or hypoplastic. Dense otic bone is present at the site of the cochlea (Fig. 4C).

\section{Common Cavity Deformity}

In this condition there is no differentiation between the cochlea and the vestibule, both together forming a cystic cavity $^{3}$ (Fig. 5). This occurs due to developmental arrest at the fourth week of gestation, when the differentiation into cochlea and vestibule has not yet taken place. 


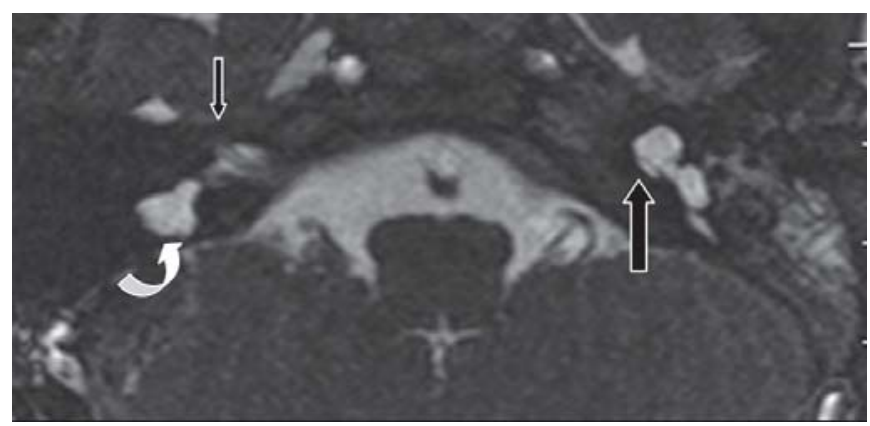

Fig. 4A: Axial 3D-FIESTA sequence shows absent right cochlea, suggesting cochlear aplasia (arrow), along with a deformed/dysplastic vestibule (curved arrow). On the left side, a dilated cystic cochlea is noted, with complete lack of modiolus

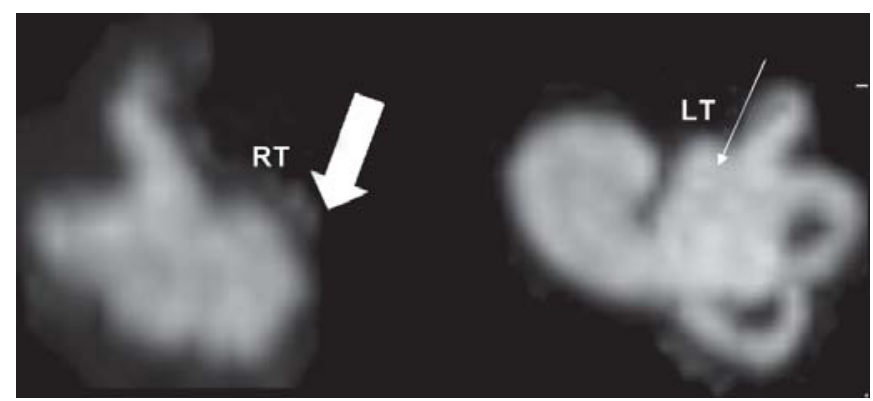

Fig. 4B: 3D MIP reconstruction of the image data set of figure 4A, clearly shows absent cochlea on the right side. On the left side a dilated cochlea is noted, with a cystic vestibule, consistent with Incomplete partition type I deformity

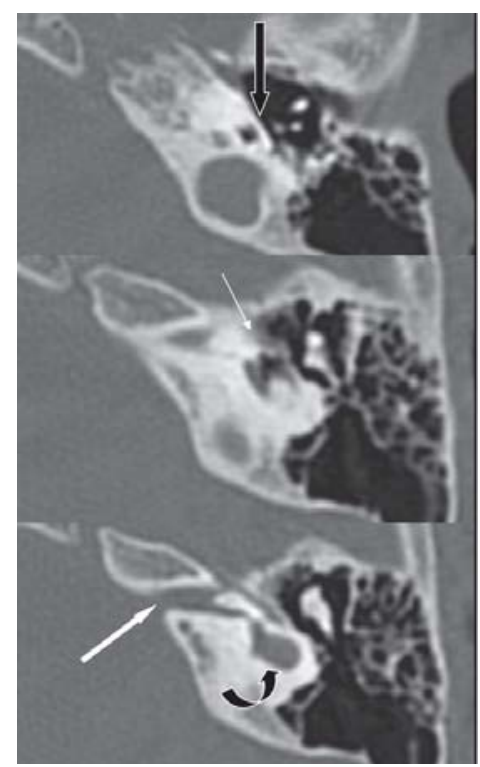

Fig 4C: HRCT axial scan at three consecutive levels shows cochlear aplasia (white arrow), with loss of cochlear promontory bulge (block black arrow), normally produced by the basal turn of cochlea. At a higher level, a small IAC is noted (white arrow) along with a dysplastic cystic vestibule (curved arrow)

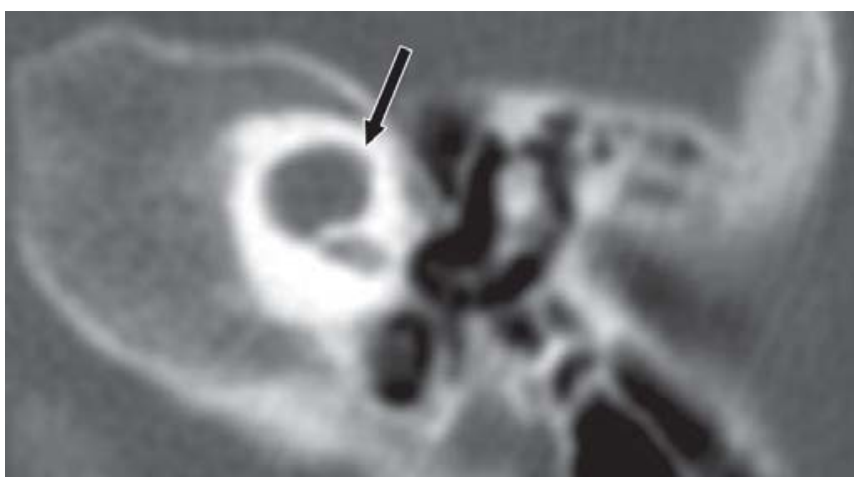

Fig. 5: HRCT axial scan showing common cavity (arrow), in which there is no differentiation between cochlea and vestibule, both forming a cystic cavity

\section{Incomplete Partition Type I (IP-I)}

In this condition, the cochlea lacks the entire modiolus and the cribriform area and appears cystic, along with a large cystic vestibule ${ }^{3}$ (Fig. 6). The dimensions of the cochlea and the vestibule are normal but the internal architecture, including the modiolus, is missing, giving it an empty cystlike appearance (Figs 4A and B; left ear). The modiolus is completely absent in its entire length from the base to the apex. One needs to note the striking difference from the IP-II (Mondini) deformity, where only the middle and the apical turns form a cystic cavity due to fusion. This pathology represents a form of common cavity that is one step more organized and differentiated than the previously described common cavity malformation, with the developmental arrest occurring in the fifth week. ${ }^{3}$

\section{Cochleovestibular Hypoplasia}

In this group, the cochlear and the vestibular structures are separate from each other and more differentiated than in IP-II, with failure of development occurring in the sixth week. ${ }^{3}$ Both the cochlea and the vestibule are small in size, with the hypoplastic cochlea seen as a small bud, coming off the IAC. ${ }^{3}$

\section{Incomplete Partition Type II (IP-II) (Mondini Deformity)}

This condition represents developmental arrest occurring at a later stage than in IP-I (in the seventh week of gestation), with the size of the cochlea and vestibule appearing normal and internal organization being more developed. ${ }^{3}$ The cochlea consists of 1.5 turns, with the middle and apical turns coalescing to form a cystic apex (due to a defect in the interscalar septum), along with a dilated vestibule and enlarged vestibular aqueduct (Figs 7A and B). 


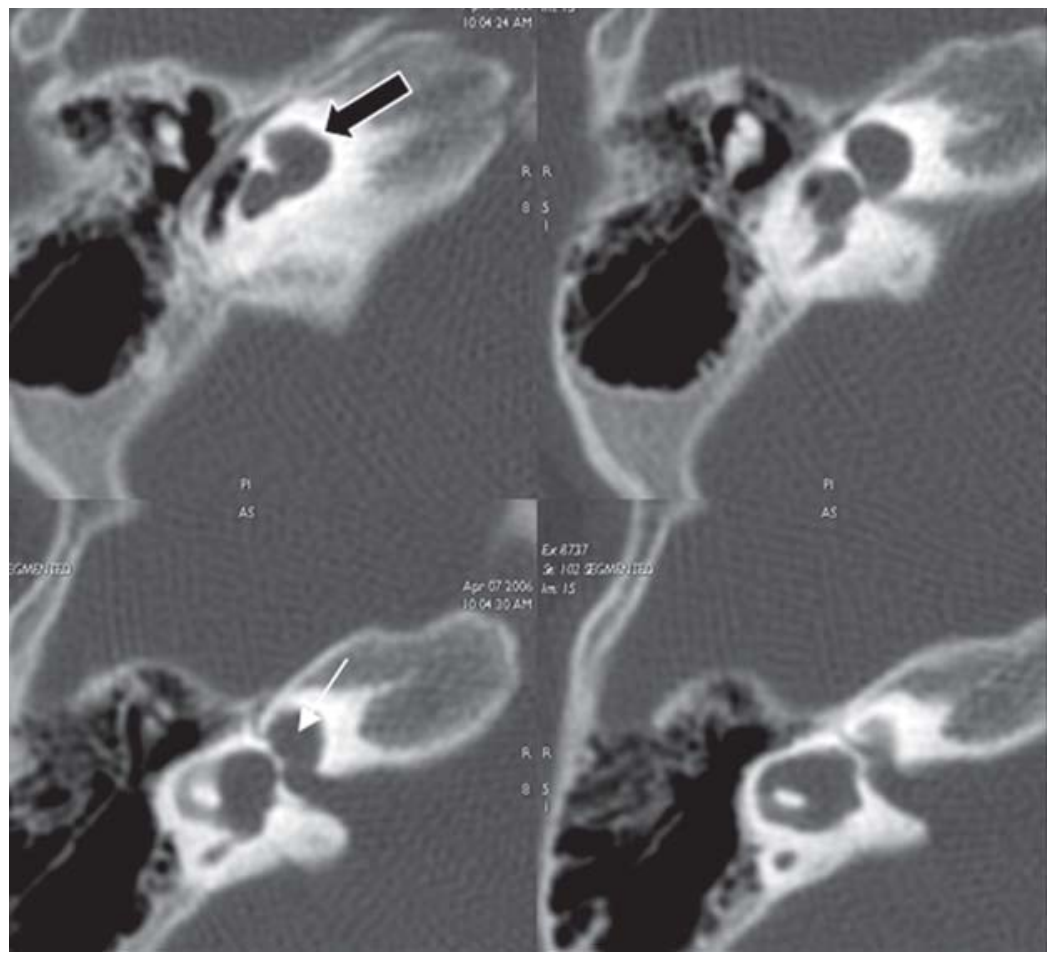

Fig. 6: HRCT axial scan shows findings of Incomplete partition type I deformity, with cystic appearance of cochlea, lacking the entire modiolus (block black arrow) and a cystic vestibule (white arrow)

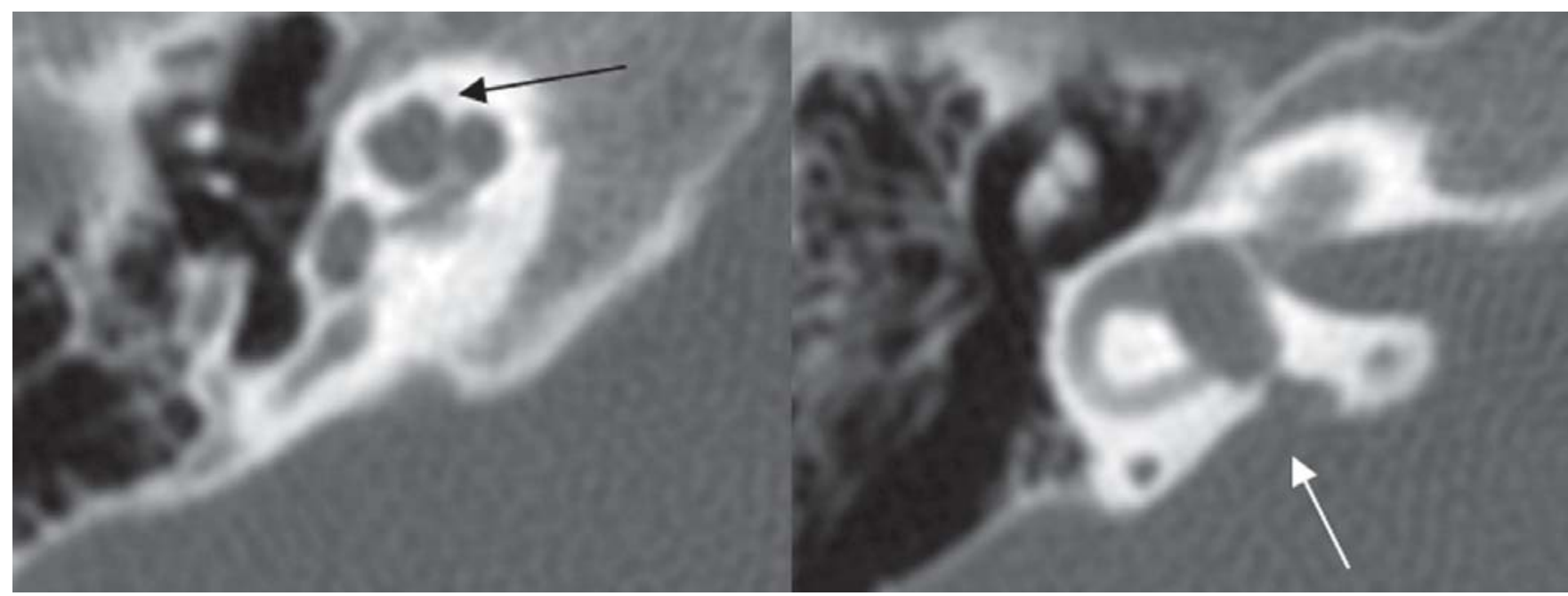

Fig. 7A: HRCT axial images show fusion of middle and apical turns of the cochlea (black arrow), prominent vestibule and dilated vestibular aqueduct (arrow), suggesting incomplete partition type II (Mondini) deformity

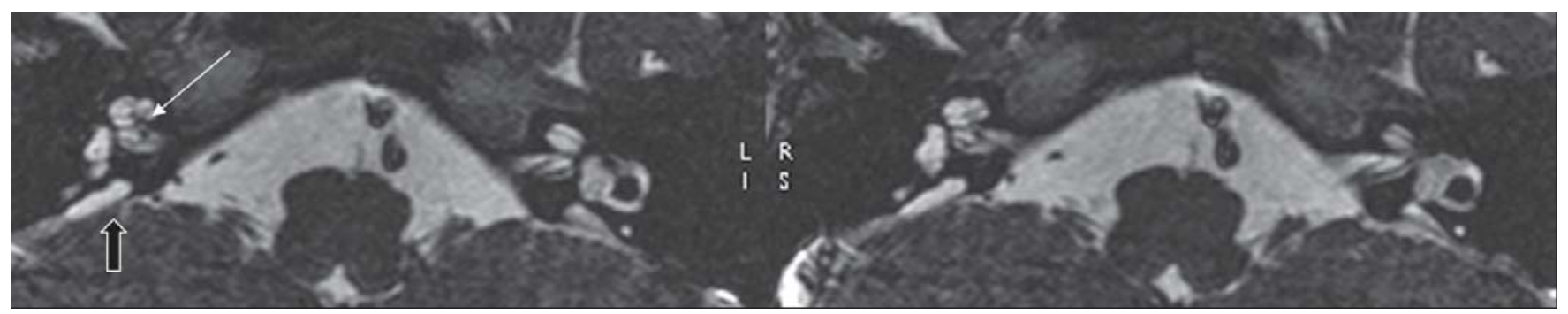

Fig. 7B: Axial 3D-FIESTA sequence shows dilated vestibular sac (block arrow) and markedly attenuated modiolus (arrow) 


\section{Dilated Vestibular Aqueduct (Fig. 8)}

This used to be thought as a finding which may occur in isolation. However, Lemmerling et al, ${ }^{12}$ in their series found that all ears with large vestibular aqueducts have an associated modiolar deficiency. Hence the term, isolated dilated vestibular aqueduct seems obsolete. IP-II (classic Mondini) is always associated with a dilated vestibular aqueduct. Isolated dilated vestibular aqueduct has also been observed and reported by other authors. ${ }^{9}$ Large vestibular aqueduct, bilaterally may be a part of a syndrome, such as Pendred syndrome and distal renal tubular acidosis, ${ }^{13}$ one needs to look for this possibility in the appropriate setting. Bamiou et al, in their series of patients with sensorineural hearing loss evaluated with HRCT, found that $60 \%$ of their patients had isolated dilated vestibular aqueduct. ${ }^{14}$ The criterion that

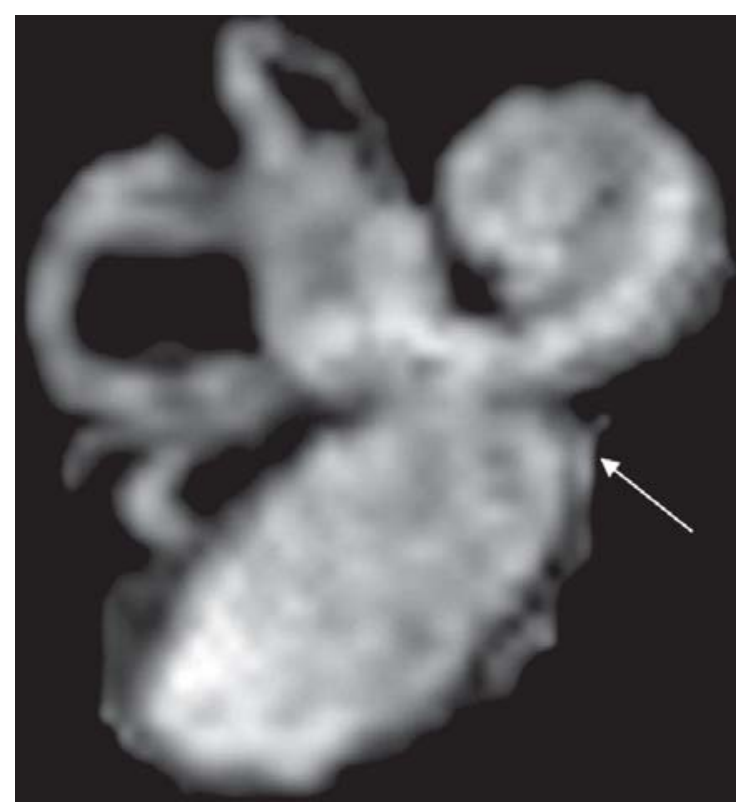

Fig. 8: 3D-MIP reconstruction of the images of another patient with IP II, well-demonstrates, a large vestibular sac (arrow) they used for a dilated vestibular aqueduct was a middle third diameter of the duct of more than $1.5 \mathrm{~mm} .{ }^{14}$

\section{Cochlear Nerve Deficiency}

We have used the term cochlear nerve deficiency to encompass both absent (Figs 3 and 9A and B) as well hypoplastic cochlear nerves, based on the study by Glastonbury et al. ${ }^{8}$ This study and another by Kim et al. ${ }^{7}$ showed that the cochlear nerve is larger than either the superior or inferior vestibular nerves in $90 \%$ of normal cases and it is of almost the same size (Fig. 2C) or larger than the facial nerve in $64 \%$ of cases.

In almost all patients with congenital cochlear nerve deficiency the IACs are small in size. As per the criteria described in literature, an abnormal IAC is diagnosed if it is $<4 \mathrm{~mm}$ in either the vertical or transverse diameter, is irregularly shaped, or is appreciably smaller than the IAC of the contralateral side. ${ }^{8}$ In the study by Valvassori et al, the IAC was found to be virtually symmetric in healthy individuals, with a difference of $<1 \mathrm{~mm}$ in $99 \%$ of patients and 1 to $2 \mathrm{~mm}$ in $1 \%$ of patients. ${ }^{9}$

\section{Isolated Cochlea}

The term isolated cochlea (Fig. 10A) is used when the cochlear aperture (which is a small canal at the fundus of the IAC (Fig. 10B), through which the cochlear nerve passes to enter the cochlea, is absent and filled with bone. ${ }^{8}$ This has also been described as hypoplasia of the bony canal for the cochlear nerve (Fig. 10C). ${ }^{10}$

\section{Attenuated Modiolus}

This may be an isolated finding or may be seen in association with other malformations. Modiolus (taken from the latin word 'hub of a wheel') is an irregular conical (or trapezoidal)-shaped porous bone within the cochlea from which the osseous spiral lamina of the cochlea projects out, which supports the organ of Corti (Fig. 2B).

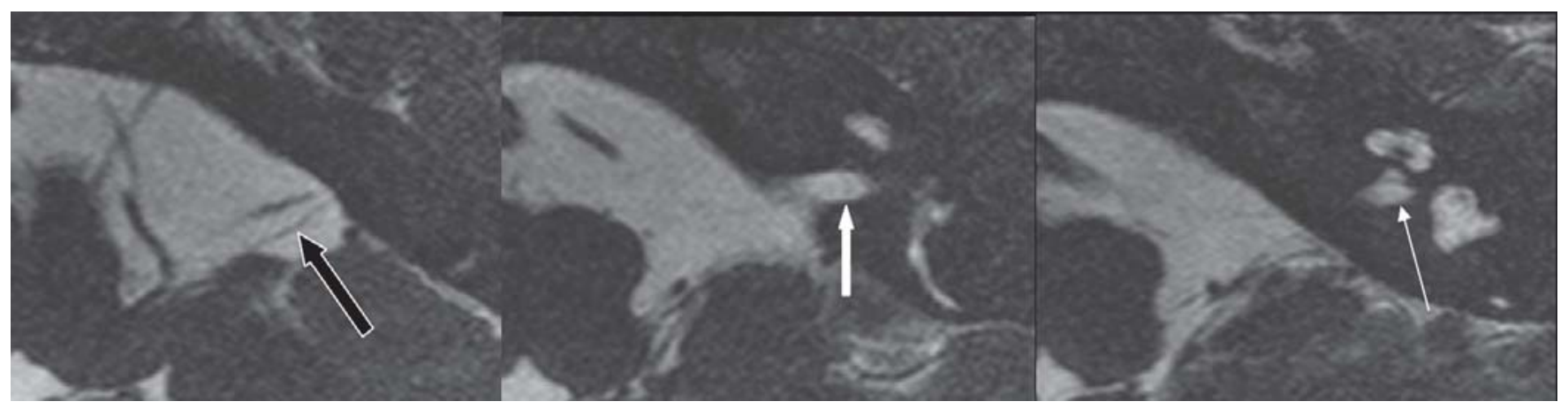

Fig. 9A: Axial 3D-FIESTA sequence shows markedly thinned-out eighth nerve in the CP angel cistern (black arrow), which is barely seen in the distal portion of the canal and the left cochlear nerve is not visualized in its normal location (arrow) 


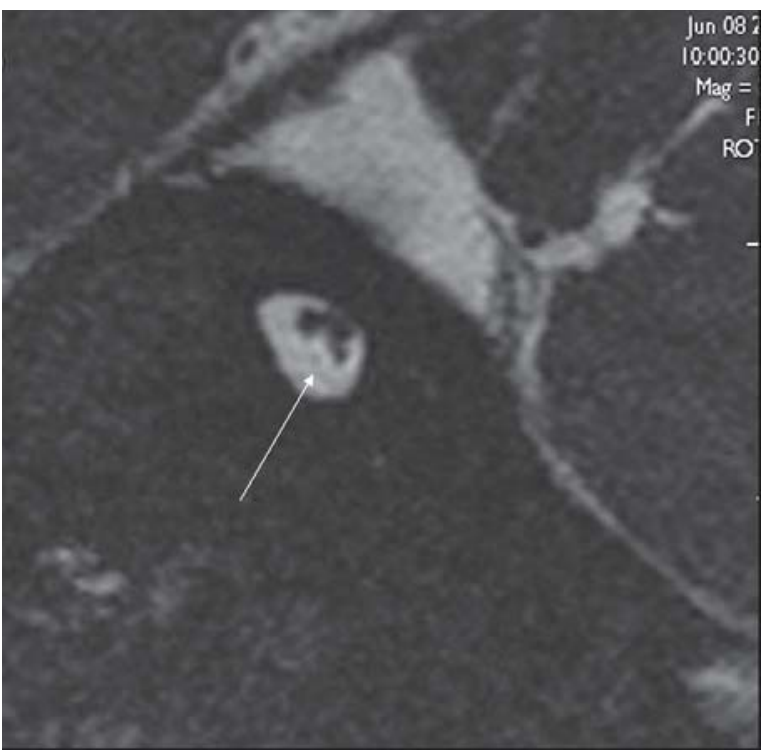

Fig. 9B: Oblique sagittal 3D-FIETSA sequence shows the facial and vestibular nerves in their normal location, with the cochlear nerve barely or almost not seen in the anteroinferior quadrant (arrow)
We label a modiolus as attenuated on the basis of subjective criteria (Fig. 11), although the more accurate method is the measurement of its area at the point of its maximum size. According to Naganawa et al, the size of the modiolus on MRI in normal volunteers is about $4.0 \mathrm{~mm}^{2} .^{11}$

In all patients, who are being evaluated for congenital sensorineural hearing loss, it is important to study brain MRI, as sometimes there may be findings of congenital TORCH infection on T2-wieghted MRI of brain showing multiple ill-defined hyperintense lesions predominantly in the cerebral subcortical white matter (Fig. 12).

\section{OTOSPONGIOSIS (OTOSCLEROSIS)}

This is an otodystrophy of the bony labyrinth and stapes, where the normal endochondral bone of the otic capsule is replaced by disorganized foci of Haversian bone. ${ }^{15} 2$ types have been described : Fenestral and Cochlear (rare, and associated with fenestral), the latter causing sensorineural hearing loss. The early stage is seen as a

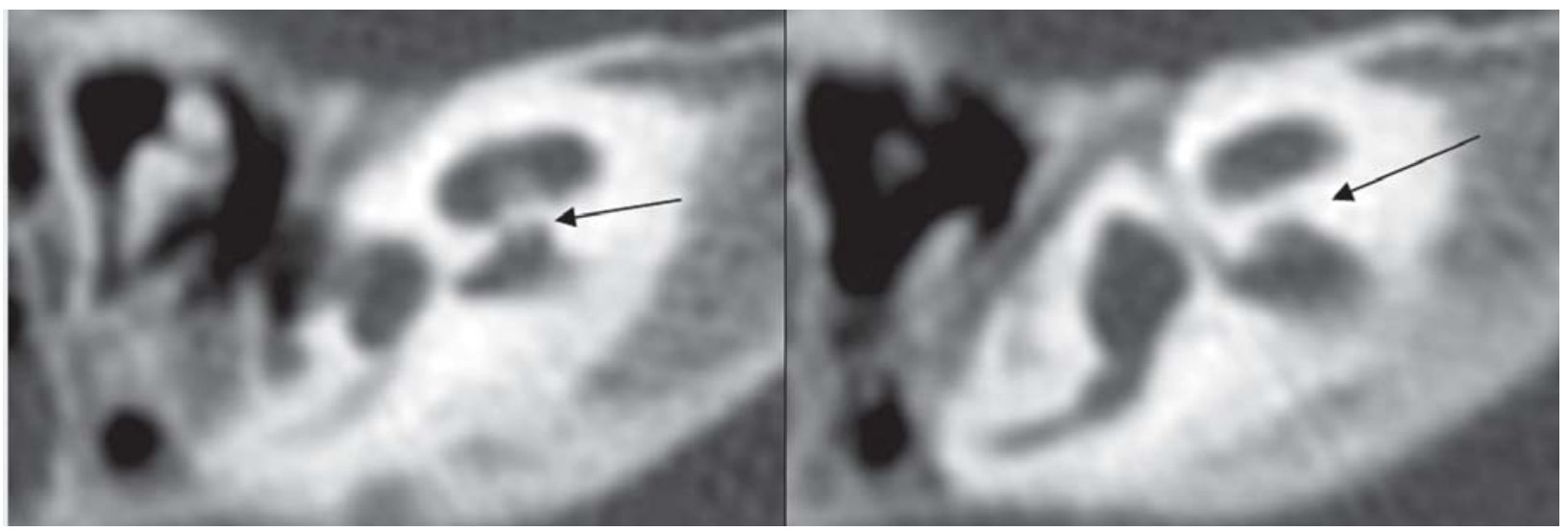

Fig. 10A: Axial HRCT shows a thick bony bar at the cochlear aperture (arrow), suggesting an isolated cochlea

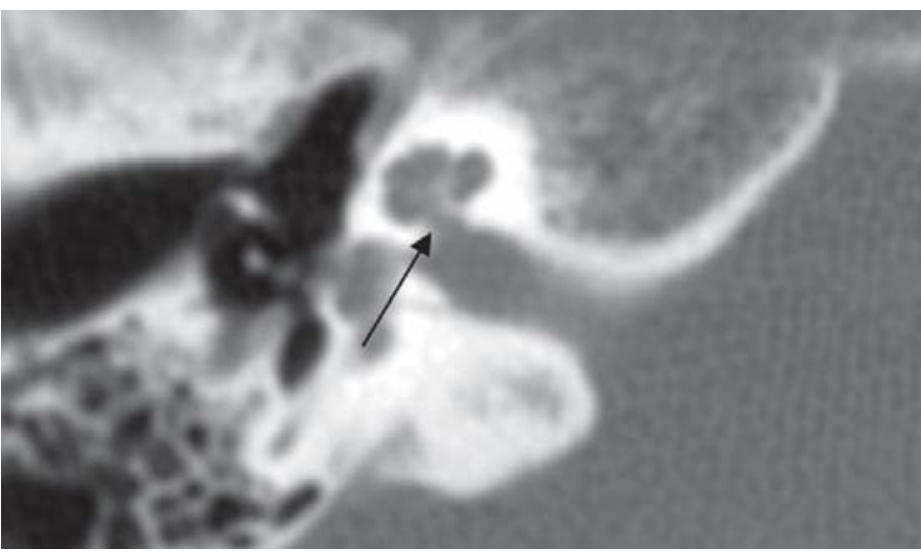

Fig. 10B: Axial HRCT shows normal appearance of the cochlear aperture at the site of the entry of the cochlear nerve, near the modiolus

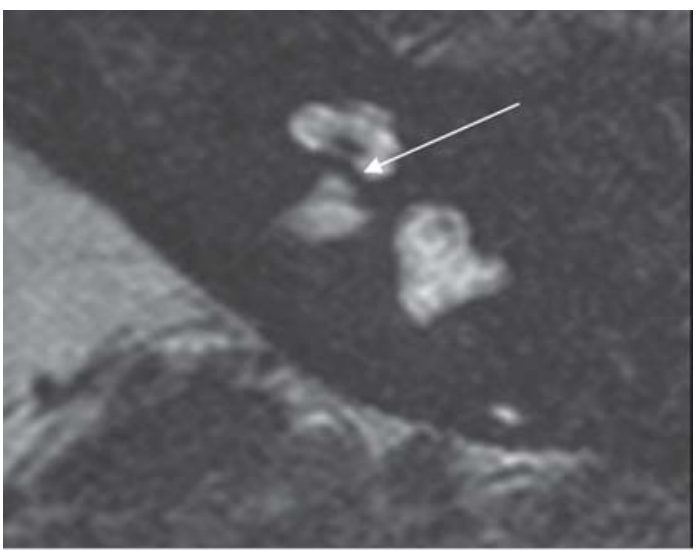

Fig. 10C: 3D-FIESTA axial sequence shows a bony bar (arrow) at the cochlear aperture (isolated cochlea). Also note left cochlear nerve deficiency 


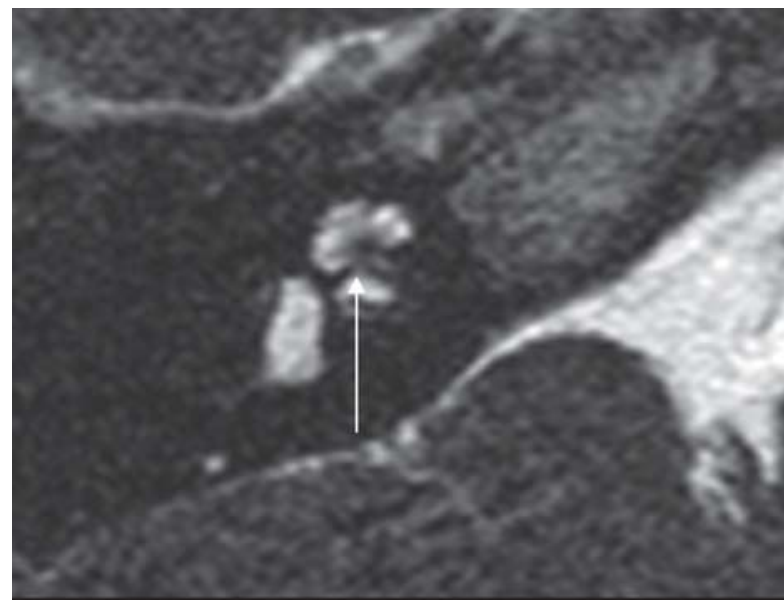

Fig. 11: Axial 3D-FIESTA sequence shows attenuated modiolus on the right side (arrow). Compare with normal modiolus in figure 2B

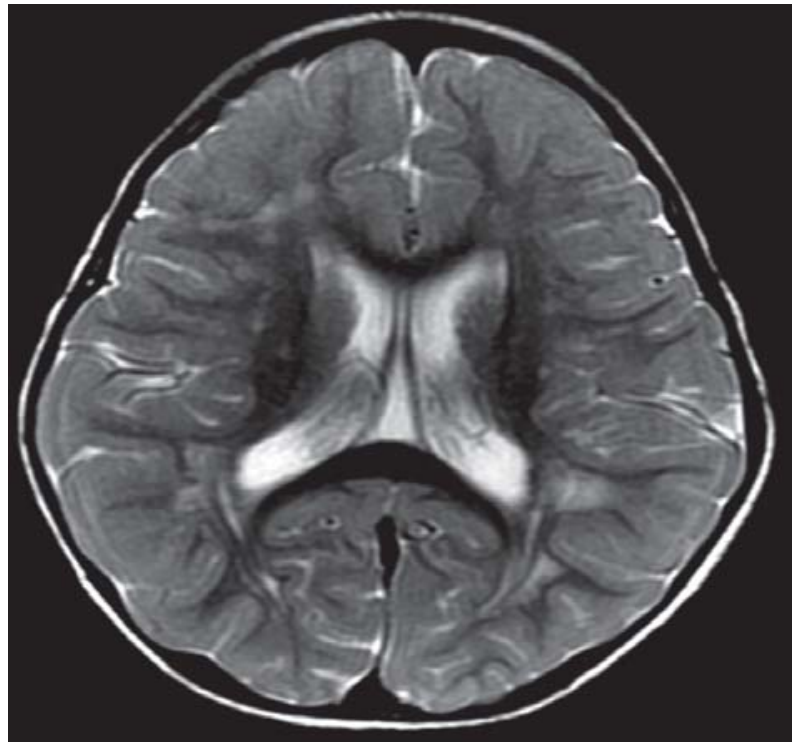

Fig. 12: MRI T2-weighted axial image shows multifocal ill-defined subcortical lesions in both cerebral white matter, most likely sequelae of $\mathrm{TORCH}$ infection

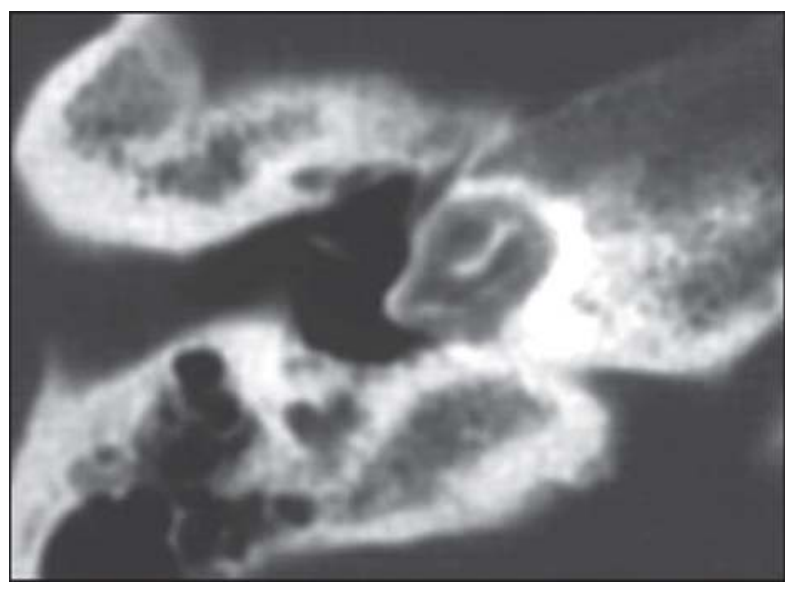

Fig. 13: Axial HRCT shows extensive lucency around all the visualized cochlear turns, consistent with both fenestral and cochlear otospongiosis also called otospongiosis lucency (otospongiosis stage) around the cochlea on HRCT (Fig. 13). In later stage there is recalcification into dense ossific plaque (otosclerosis stage). MRI may show enhancement on $\mathrm{T} 1$, in the otic capsule in the early stage and also pericochlear T2 hyperintense signal. ${ }^{15}$

\section{INFLAMMATORY-INFECTIVE}

With the advent of higher resolution and better imaging techniques on MRI, subtle abnormalities of inner ear or eighth cranial nerve are more easier to identify, such as in acute labyrinthitis, where enhancement of the membranous labyrinth can be seen on postgadolinium T1-weighted images. In infectious or autoimmune labyrinthitis, there is a break in the blood-labyrinthine-barrier, secondary to inflammation, resulting in enhancement of the cochlea and/ or vestibule on MRI. ${ }^{2}$ In later stages disease may progress to ossification or labyrinthitis ossificans, where diffuse T2 hypointense signal is seen on T2, better appreciated on T2 gradient sequence such as FIESTA . This stage of ossicans is well seen on HRCT of temporal bones (Fig. 14), however the fibrotic phase will not be readily appreciated on HRCT, which is seen well on 3D gradient MRI sequence (Fig. 15).

Hemorrhagic labyrinthitis is also well seen as hyperintense signal in the membranous labyrinth on precontrast T1 weighted sequence on MRI (Fig. 16). Vestibular neuritis may be seen on MRI as enhancement of the eighth nerve, and should not be mistaken for a small vestibular Schwannoma, where enhancement is focal and nodular.

Other conditions that may cause SNHR due to infection or inflammation are leptomeningitis (tuberculous, bacterial or fungal), and neurosarcoidosis, which may show linear or nodular enhancement along the eighth nerve.

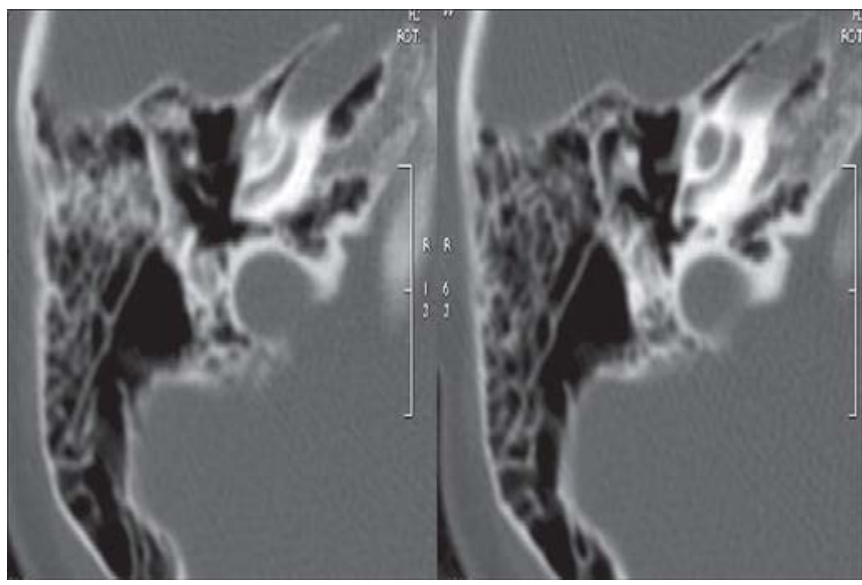

Fig. 14: Axial HRCT shows ossificans along the margin of the basa turn of cochlea consistent with labyrinthitis ossificans 


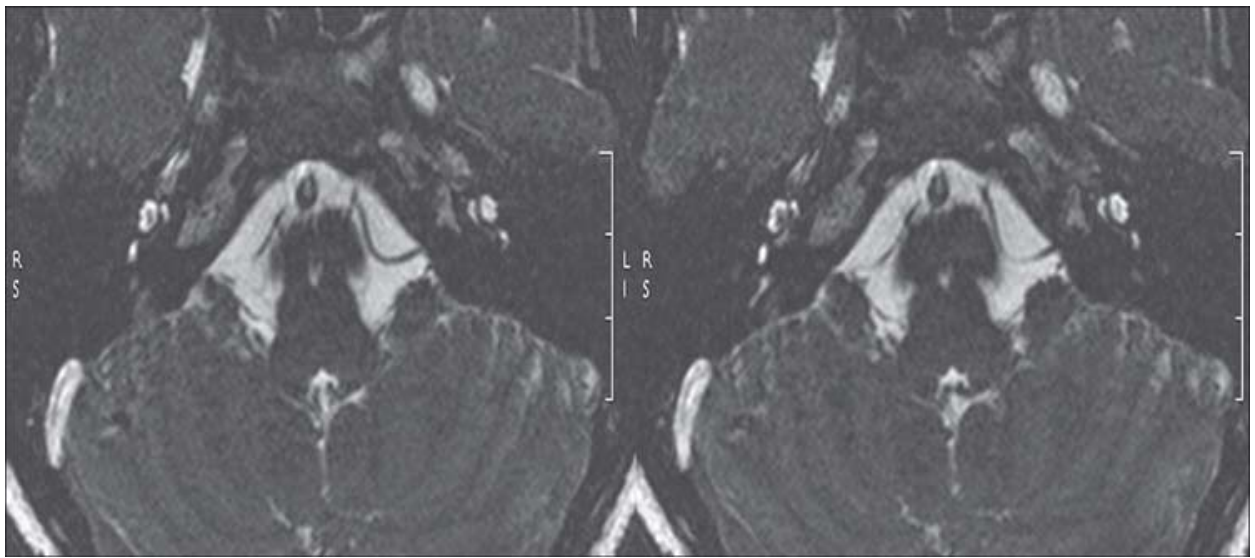

Fig. 15: 3D-FIESTA axial MRI in early fibrotic phase of labyrinthis ossificans shows T2 hypointense signal along the posterior margin of the basal turns of both cochlea

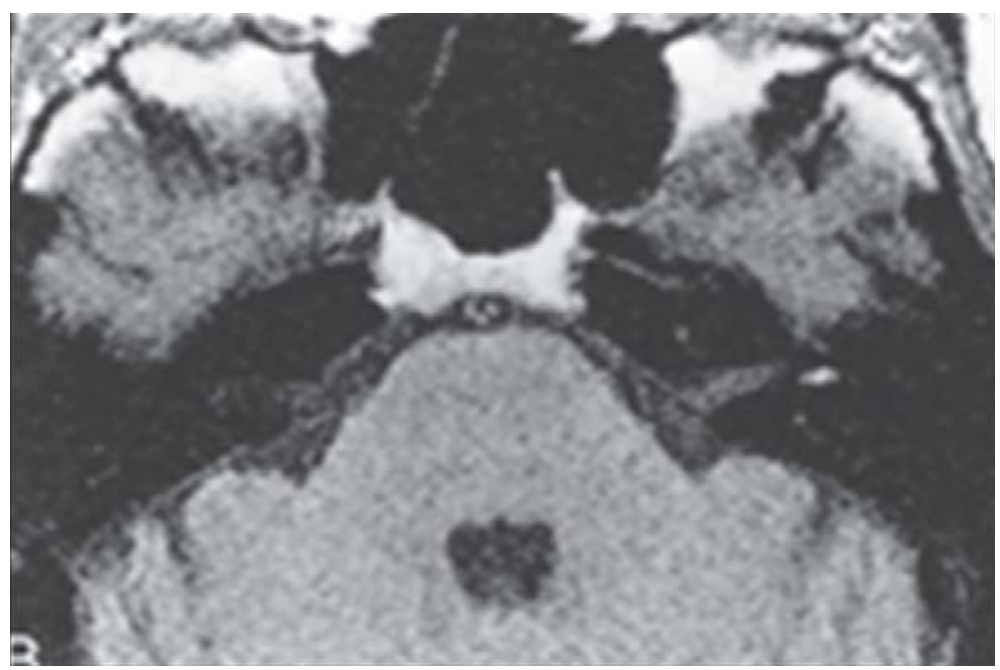

Fig. 16: Precontrast T1-weighted axial MRI shows hyperintensity in the left cochlea due to hemorrhagic labyrinthitis

\section{POSTERIOR FOSSA TUMORS}

\section{Acoustic Neuroma}

Most common mass encountered in the IAC (internal auditory canal) and CPA (cerebello-pontine angle) cistern is acoustic neuroma which is a benign slow growing tumor arising from Schwann cells along the VIII cranial nerve, comprising approximately $85 \%$ of CPA masses. ${ }^{2}$ These most commonly arise from vestibular nerve at the area of the Scarpa ganglion in the IAC. These are best visualized on MRI, where an ovoid shape mass is seen contained within the IAC (which may be enlarged) into the CPA cistern. At times one may see bilateral acoustic Schwannomas which confirms the diagnosis of neurofibromatosis type II (Fig. 17). At times these may be isolated to IAC, without extension into the CPA cistern (Fig. 18). These are usually isointense on T1-weighted sequence and hyperintense on T2, with diffuse enhancement on postcontrast scans. Tumors which are larger in size may show heterogeneous signal on $\mathrm{T} 2$ and heterogenous enhancement with cyst like areas. Hemorrhage may also be seen but is rare, and is visible as hyperintense signal on precontrast T1-weighted sequence. Calcification may also occur rarely. CT will show enlarged IAC in case of large tumors. CT is useful to see calicification if any. However, small lesions at times can be missed even on contrast-enhanced CT and MRI is the preferred modality of choice to evaluate posterior fossa tumors in general.

\section{Mengiomas}

Approximately 3 to $8 \%$ of CPA masses and may look similar to acoustic neuromas. ${ }^{2}$ However, these generally have a broad-based dural attachment in the posterior fossa, with dural tail-like enhancement (Fig. 19) on the postgadolinium scan, which distinguishes them from acoustic neuromas. 


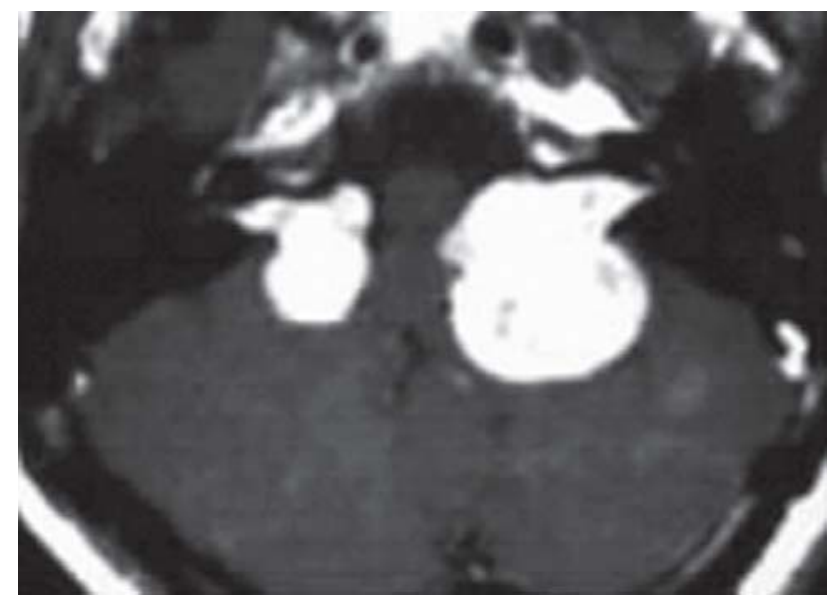

Fig. 17: MRI axial contrast enhanced T1-weighted image shows bilateral large enhancing $\mathrm{CP}$ angle masses extending into both the IAC, consistent with acoustic Schwannomas, in a case of neurofibromatosis type II

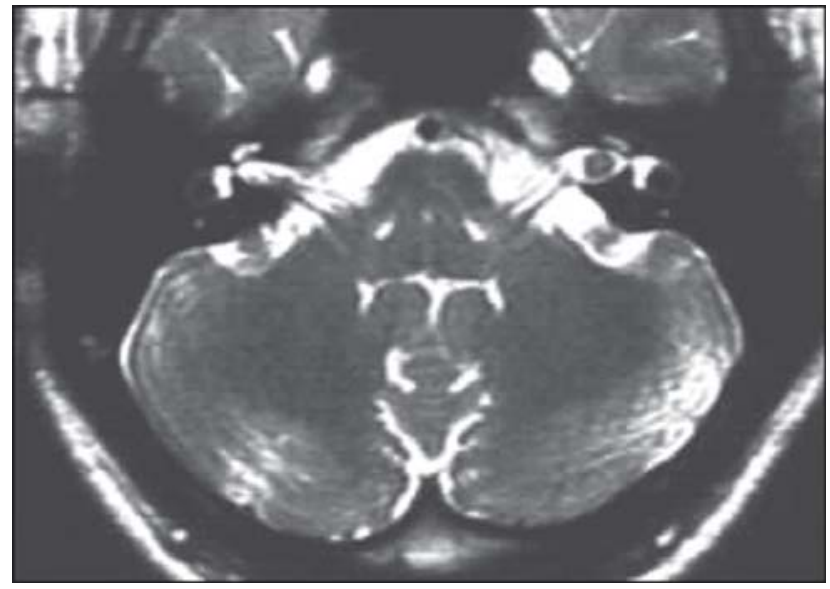

Fig. 18: Thin T2-weighted axial sequence shows a small hypointense nodule/Schwannoma, in the left IAC

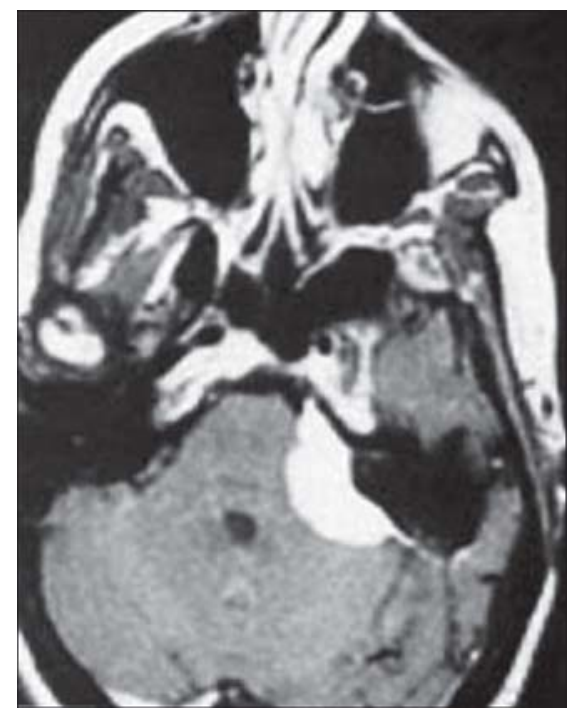

Fig. 19: Postcontrast T1-weighted axial MRI shows a diffusely enhancing left CP angle mass lesion with a broad base and adjacent linear dural enhancement typically seen in a meningioma
Meningiomas tend to be hypointense on T2 due to increased celluarity and diffuse calcification, the latter better appreciated on CT scan. On CT they may show diffuse mild hyperdensity or frank calcification on the plain scan. Sometimes meningiomas extend into the IAC, although they are usually located eccentrically to the porus.

Other tumors: Other rare tumors in the posterior fossa, in the region of IAC or CPA cistern attributable to sensorineural hearing loss are metastasis (especially from breast or lung), lymphoma, melanoma, endolymphatic sac tumors.

\section{OTHERS}

Vestibular neuritis may be seen as diffuse enhancement of the eighth nerve on postcontrast MRI, which will be smooth and diffuse and should not be mistaken for small vestibular Schwannoma, where the enhancement is focal and nodular.

Superficial siderosis due to repeated subarachnoid hemorrhage may cause hemosiderin deposition on the surface of cerebellum, brainstem and the eighth nerve producing typical marked T2 hypointense signal on MRI, also well seen with blooming on gradient sequence.

Vascular causes of sensorineural hearing loss include anterior inferior cerebellar artery infarct, small arterio-venous malformation, rarely a vascular loop significantly compressing the eighth nerve.

Temporal bone trauma causing SNHL with fractures across the inner ear structures can also be seen well on HRCT (Fig. 20).

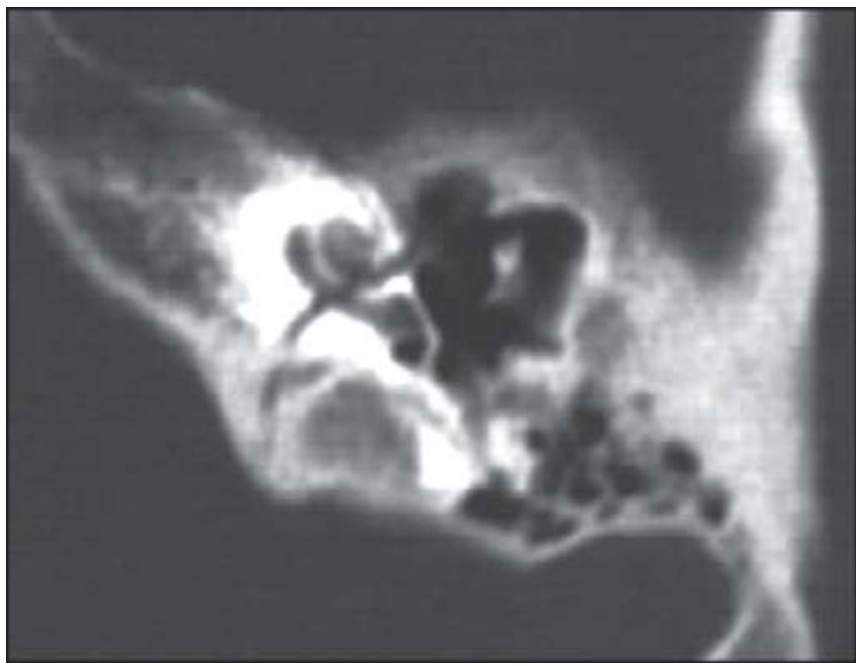

Fig. 20: Axial HRCT shows a transverse fracture across the left cochlea, in a patient with post-traumatic profound sensorineural hearing loss 


\section{CONCLUSION}

Imaging plays an important role in diagnosis and work-up of patients with SNHR. There are a whole lot of etiologies for SNHR, many of which have typical findings on HRCT and MRI, which help in differentiation of these causes and therefore guides the treating surgeon in further management. Both CT and MRI are complimentary to each other and should be judiciously used by the radiologist in diagnosing the various conditions, discussed above.

\section{ACKNOWLEDGMENTS}

I would like to acknowledge all my colleagues, especially Dr Shailendra Maheshwari and also ENT department of our institute without whom writing this article would not have been possible.

\section{REFERENCES}

1. Parry DA, Booth T, Roland PS. Advantages of magnetic resonance imaging over computed tomography in preoperative evaluation of pediatric cochlear implant candidates. Otol Neurotol 2005;26(5):976-82.

2. Michele BSM, Barry EH. Imaging of Hearing Loss: Otolaryngologic clinics of North America 2008(41);157-78.

3. Sennaroglu L, Saatci I. A new classification for cochleovestibular malformations. The Laryngoscope Lippincott Williams and Wikins, Inc., Philadelphia (C), Luxford 2002. The American Laryngological, Rhinological and Otological Society, Inc. Laryngoscope 2002;112:2230-41.

4. Gupta SS, Maheshwari SM, Kirtane MV, Shrivastav N. Pictorial review of MRI/CT Scan in congenital temporal bone anomalies, in patients for cochlear implant: Indian Journal of Radiology and Imaging 2009;19(2):99-106.
5. Jackler RK, Luxford WM, House WF. Congenital malformations of the inner ear: A classification based on embryogenesis. Laryngoscope 1987;97:2-14.

6. Curtin HD, Sanelli PC, Som PM. Temporal bone: Embryology and Anatomy Chapter (19) In: Som PM, Curtin HD. Head and Neck Imaging (4th ed). Mosby; 2003;2:1057-75.

7. Kim HS, Kim DI, Chung IH, Lee WS, Kim KY. Topographical relationship of the facial and vestibulocochlear nerves in the subarachnoid space and internal auditory canal. AJNR Am J Neuroradiol 1998;19:1155-61.

8. Glastonbury CM, Davidson HC, Harnsberger HR, Butler J, Kertesz TR, Shelton C. Head and Neck. Imaging Findings of Cochlear Nerve Deficiency. American Journal of Neuroradiology 2002 April;23:635-43.

9. Valvassori GE, Pierce RH. The normal internal auditory canal. AJR Am J Roentgenol 1964;92:773-81.

10. Fatterpekar GM, Mukherji SK, Alley J, Lin Y, Castillo M. Hypoplasia of the bony canal for the cochlear nerve in patients with congenital sensorineural hearing loss: Initial observations. Radiology 2000;215:243-46.

11. Naganawa S, Ito T, Iwayama E, Fukatsu H, Ishigaki T, Nakashima T, and Ichinose N. Head and Neck Imaging. MR Imaging of the Cochlear Modiolus: Area Measurement in Healthy Subjects and in Patients with a Large Endolymphatic Duct and Sac. Radiology 1999;213:819-23.

12. Lemmerling MM, Mancuso AA, Antonelli PJ, Kubilis PS. Normal modiolus: CT appearance in patients with a large vestibular aqueduct. Radiology 1997;204:213-19.

13. Berrittini S, Forli F, Bogazzi F, et al. Large vestibular aqueduct syndrome: Audiological, radiological, clinical and genetic features American Journal of otolaryngology Head and neck Medicine and Surgery 2005:26 363-71.

14. Bamiou DE, Phleps P, Sirimanna T. Temporal bone computed tomography findings in bilateral sensorineural hearing loss. Arch Dis Child 2000;82:257-60.

15. Goh JPN, Chan LL, Tan TY. MRI of cochlear otosclerosis British Journal of Radiology 2002(75);502-05. 